\title{
Thermal ion measurements on board Interball Auroral Probe by the Hyperboloid experiment
}

\author{
N. Dubouloz ${ }^{1}$, J.-J. Berthelier ${ }^{1}$, M. Malingre ${ }^{1}$, L. Girard ${ }^{1 *}$, Y. Galperin ${ }^{2}$, J. Covinhes ${ }^{1}$, D. Chugunin ${ }^{2}$, M. Godefroy ${ }^{1}$, \\ G. Gogly ${ }^{1}$, C. Guérin ${ }^{1}$, J.-M. Illiano ${ }^{1}$, P. Kossa ${ }^{1}$, F. Leblanc ${ }^{1}$, F. Legoff ${ }^{1}$, T. Mularchik ${ }^{2}$, J. Paris ${ }^{1}$, W. Stzepourginski ${ }^{1}$, \\ F. Vivat ${ }^{1}$, L. Zinin ${ }^{3}$ \\ ${ }^{1}$ Centre d'étude des Environnements Terrestre et Planétaires, Centre National de la Recherche Scientifique, 4 avenue de Neptune, \\ F-94107 Saint-Maur Cedex, France; Tel: + 1451142 62; Fax: + 1488944 33; e-mail: Nicolas.Dubouloz@cetp.ipsl.fr \\ ${ }^{2}$ Space Research Institute, Russian Academy of Science, Profsoyuznaya str., 84/32, GSP-7, Moscow 117810, Russia \\ ${ }^{3}$ University of Kaliningrad, Kaliningrad, Russia
}

Received: 25 August 1997 / Revised: 04 May 1998 / Accepted: 04 May 1998

\begin{abstract}
Hyperboloid is a multi-directional mass spectrometer measuring ion distribution functions in the auroral and polar magnetosphere of the Earth in the thermal and suprathermal energy range. The instrument encompasses two analyzers containing a total of 26 entrance windows, and viewing in two almost mutually perpendicular half-planes. The nominal angular resolution is defined by the field of view of individual windows $\approx 13^{\circ} \times 12.5^{\circ}$. Energy analysis is performed using spherical electrostatic analyzers providing differential measurements between 1 and $80 \mathrm{eV}$. An ion beam emitter (RON experiment) and/or a potential bias applied to Hyperboloid entrance surface are used to counteract adverse effects of spacecraft potential and thus enable ion measurements down to very low energies. A magnetic analyzer focuses ions on one of four micro-channel plate (MCP) detectors, depending on their mass/charge ratio. Normal modes of operation enable to measure $\mathrm{H}^{+}, \mathrm{He}^{+}, \mathrm{O}^{++}$, and $\mathrm{O}^{+}$simultaneously. An automatic MCP gain control software is used to adapt the instrument to the great flux dynamics encountered between spacecraft perigee $(700 \mathrm{~km})$ and apogee $(20000 \mathrm{~km})$. Distribution functions in the main analyzer half-plane are obtained after a complete scan of windows and energies with temporal resolution between one and a few seconds. Three-dimensional (3D) distributions are measured in one spacecraft spin period $(120 \mathrm{~s})$. The secondary analyzer has a much smaller geometrical factor, but offers partial access to the 3D dependence of the distributions with a few seconds temporal resolution. Preliminary results are presented. Simultaneous, local heating of both $\mathrm{H}^{+}$and $\mathrm{O}^{+}$ions resulting in conical distributions below $80 \mathrm{eV}$ is observed up to 3 Earth's radii altitudes. The thermal ion signatures associated with large-scale nightside magne-
\end{abstract}

Correspondence to: $\mathrm{N}$. Dubouloz

${ }^{*}$ L. Girard now at Centre National d'Etudes en Télécommunications, France Telecom, 38-40 avenue du Général Leclerc, F-92794 Issy Cedex, France tospheric boundaries are investigated and a new ion outflow feature is identified associated to the polar edge of the auroral oval. Detailed distribution functions of injected magnetosheath ions and ouflowing cleft fountain ions are measured down to a few eVs in the dayside.

Key words. Ionosphere (auroral ionosphere; particle acceleration; ionosphere-magnetosphere interactions)

\section{Introduction and scientific objectives}

Space missions conducted over the last 15 years have led to the gradual understanding that low-energy (below a few hundreds of eV) plasmas significantly contribute to the processes which govern the coupling between the Earth's ionosphere and magnetosphere. As a matter of fact, thermal and slightly suprathermal plasma often represents the dominant contribution to the local number and/or energy density, and therefore strongly drives the global dynamics of the ionoshperic and magnetospheric system, especially in the ideal MHD context of frozen-in magnetic field lines. Moreover, thermal plasma supports the propagation of electrostatic and electromagnetic waves, and in some cases provides the necessary free energy for microscopic instabilities.

It is now widely admitted that a significant amount of the magnetospheric plasma is of ionospheric, and not solar wind, origin. First indirect evidence of ionospheric particles escaping along magnetic field lines was the discovery of energetic $\mathrm{O}^{+}$ions in the magnetosphere (Shelley et al., 1972). The existence of low-energy $\mathrm{H}^{+}$ and $\mathrm{He}^{+}$outflows was supported in the 1970s by the Explorer 31 (Hoffman, 1970), OGO 2 (Taylor and Walsh, 1972), and ISIS 2 (Hoffman et al., 1974) spacecrafts. Direct quantitative measurements of the 
characteristics of these outflows were obtained during the Dynamics Explorer (DE) and Akebono missions. They revealed a rather complex picture of ion escape processes, involving various regimes associated with more or less specific geophysical regions. Because these escape processes are most commonly observed at high geomagnetic altitudes, they are often referred to as "polar wind", without distinction. A review of theoretical, numerical, and experimental studies of polar wind can be found in Ganguli (1996). In the polar cap, supersonic $\mathrm{H}^{+}$and $\mathrm{He}^{+}$outflows are frequently observed (Nagai et al., 1984). Their properties are reminiscent of the "classical" polar wind model, in which field-aligned motion is driven by pressure gradients and by the ambipolar electric field (Banks and Holzer, 1969). Classical polar wind theory, however, is not able to explain other ion outflows characterized by temperatures much larger than typical ionospheric temperatures, or by the presence of heavy (mainly $\mathrm{O}^{+}$) ions also escaping along the field lines (Gurgiolo and Burch, 1982; Green and Waite, 1985). Such processes are often observed in auroral regions, and obviously require a complementary acceleration mechanism. Many theoretical and numerical models have been developed in order to explain these "generalized" polar wind phenomena. They include various causes of ion upward acceleration, such as heating by wave-particle interactions or Joule effect, and hot photoelectrons or magnetospheric electrons inducing enhanced ambipolar electric fields. The horizontal plasma dynamics associated to magnetospheric convection is a further factor acting on ion outflows. First, convection transports the magnetic field lines across the auroral and polar regions; as a result, outflows along these field lines experience time-dependent boundary conditions which strongly affect their characteristics (Schunk and Sojka, 1989). At low altitudes, the relative horizontal drift between ions and neutrals also produces significant frictional heating leading to additional upward acceleration (St-Maurice and Schunk, 1979). Finally, $\mathbf{E} \times \mathbf{B}$ convection contributes to $\mathrm{O}^{+}$acceleration at higher altitudes where field lines have smaller curvature radii; this effect can be described as centrifugal acceleration in the convecting plasma frame of reference (Cladis, 1986). The respective role of the many physical processes involved in ionospheric outflows has still to be determined; this theoretical effort will need to rely on new, higher resolution thermal plasma measurements.

As already stressed, acceleration due to wave-particle interactions is thought to constitute a significant ingredient of the generalized polar wind mechanism. Several different kinds of accelerated ion distributions can be distinguished at energies up to a few hundreds of eVs. Upward field-aligned beams are frequently observed and interpreted in terms of acceleration parallel to the magnetic field line. Quasi-static parallel electric fields, electrostatic shocks generating impulsive parallel electric fields in the ULF frequency range (Mozer et al., 1980), and kinetic Alfvèn waves with a significant parallel electric component (Louarn et al., 1994) may well operate along a large part of magnetic field lines.
Conical type ion distributions have been investigated in the frame of various mechanisms involving either resonant interaction with a variety of waves - lower hybrid (Retterer et al., 1994), electrostatic ion cyclotron (Ungstrup et al., 1979), electromagnetic ion cyclotron (Rauch et al., 1993; Le Quéau et al., 1993) - or nonresonant interactions through stochastic acceleration by broadband fluctuating fields below the $\mathrm{O}^{+}$ion gyrofrequency (Lundin et al., 1990). It must be stressed that these mechanisms are not mutually exclusive; indeed, low-energy ion measurements provide key information in order to disentangle the effects of competing waveparticle interactions (André et al., 1994).

The results accumulated over the years have therefore imposed low-energy plasma measurements as a major goal of magnetospheric satellite missions, and enlightened the need of dedicated experiments. On board the Interball Auroral Probe (IAP), this task is devoted to the Hyperboloid experiment, which is an ion mass spectrometer aimed at measuring the low-energy threedimensional (3D) distributions of the four "major" ions $\mathrm{H}^{+}, \mathrm{He}^{+}, \mathrm{O}^{++}$, and $\mathrm{O}^{+}$. Its $0-80-\mathrm{eV}$ energy range is suited to studies of processes involving thermal and slightly suprathermal ions in the Earth's auroral and polar regions at altitudes of 700 to $20000 \mathrm{~km}$ covered by Interball AP. By some aspects, Hyperboloid derives from the Dyction instrument which was previously flown on Aureol-3 (Berthelier et al., 1982).

A major goal of Hyperboloid is to contribute to a more comprehensive classification of observed ion outflow regimes, in order to associate them with the various theoretical and numerical models. With this respect, Hyperboloid provides detailed ion distribution function measurements, especially in the 10 000-20 000$\mathrm{km}$-altitude range which has remained poorly explored before Interball AP launch. The interpretation of Hyperboloid data will benefit from the simultaneous activity of other plasma experiments, e.g., Thermal Ion Dynamics Experiment (TIDE) (Moore et al., 1995), and Toroidal Imaging Mass-Angle Spectrograph (TIMAS) (Shelly et al., 1995), both on board the Polar satellite orbiting at higher altitudes with an apogee of 9 Earth's radii. Comparing the observed outflow properties at different altitudes, especially at the occasion of magnetic conjugations, is expected to shed new light on polar wind theory. Observations by ground-based radars (e.g., EISCAT, Super-DARN) should also complement this new, more global experimental picture of polar wind.

It has already been highlighted that magnetospheric convection is one of the factors influencing outflow processes. In the regions which are explored by Interball $\mathrm{AP}$, the knowledge of ion motion perpendicular to the magnetic field is of particular interest since it governs the ultimate fate of the extracted ions. This is especially important in the cusp region where the structure and intensity of convection closely depends on the orientation and intensity of the interplanetary magnetic field and on the solar wind velocity. Hyperboloid measurements of horizontal velocities and DC electric fields from IESP (Perraut et al., 1998) will complement each other in order to provide a quantitative picture of 
convection at Interball AP altitude. Owing to the $62.8^{\circ}$ inclination of the orbit plane, the satellite practically travels along L-shells near apogee, thus enabling the monitoring of the convection at almost constant auroral latitudes during extended periods. This capability, associated with the simultaneous observations of ionospheric plasma flows by the ESR and EISCAT radars, and by the Super-DARN network of coherent radars, will result in unprecedented data sets to study the solar wind-magnetosphere-ionosphere coupling: satellite data, which give access to small-scale $(\approx 1$ to $10 \mathrm{~km})$ structures such as arcs or vortices (which might be associated to flux transfer events at the magnetopause), will greatly benefit from the knowledge of the larger scale $(\approx 50 \mathrm{~km})$ and slower $(\approx 1 \mathrm{~min})$ variations measured from the ground.

At Interball AP altitudes, the distinction between the different types of accelerated ion distributions becomes more difficult due to the magnetic mirror force tending to fold-up conical distributions which ultimately look like beams. However, the use of the full angular and energy resolution of Hyperboloid, together with the correlation with wave instruments, will provide the required data to study microscopic wave-particle interactions in detail. First, the direct knowledge of thermal plasma parameters will enable a reliable determination of wave dispersion relations and growth rates, without relying on indirect and more qualitative measurements such as density inferred from Langmuir probe data or from auroral hiss cut-off. The second expected progress will result from the possibility to measure slightly suprathermal ions, i.e. ions right at the beginning of their acceleration process, before the features of the distribution function become obscured by spatial and temporal inhomogeneities in the acceleration process. Of utmost importance is the possibility offered by Hyperboloid to obtain simultaneous low-energy distributions of $\mathrm{H}^{+}$, $\mathrm{He}^{+}, \mathrm{O}^{++}$, and $\mathrm{O}^{+}$; comparing the level of energization reached by the various ion species should provide precious clues to identify acceleration mechanisms.

\section{Instrument description}

\subsection{Specifications}

In order to fulfill the assigned scientific objectives, the Hyperboloid ion mass spectrometer was designed to measure the full 3D distribution functions of low-energy ions.

At altitudes between 700 and $20000 \mathrm{~km}$ along Interball AP orbit, and under average conditions, the major ion species are $\mathrm{H}^{+}, \mathrm{He}^{+}$, and $\mathrm{O}^{+}$. These three ions are measured almost systematically in routine modes of operation. Some specific modes can be used in order to measure $\mathrm{N}^{+}$and molecular ions (cf. Sect. 2.4). Although representing for low solar and magnetic activity a small contribution to the total density, $\mathrm{O}^{++}$ may in some conditions reach densities as high as $\mathrm{O}^{+}$, and is therefore included in the routinely measured ion species; the simultaneous measurement of $\mathrm{O}^{+}$and $\mathrm{O}^{++}$ is a precious tool to trace the effects of acceleration by DC electric fields or by resonant processes.

In the high-altitude portion of Interball AP orbits, $\mathrm{H}^{+}$is usually the major ion, total ion densities ranging between about a few $10^{-2}$ and a few tens of $\mathrm{cm}^{-3}$. The investigation of such dilute plasmas requires a very high sensitivity in order to detect differential fluxes down to about a few $10^{3}\left(\mathrm{~cm}^{2} \mathrm{~s} \mathrm{sr} \mathrm{eV}\right)^{-1}$. Moreover, the energy bandwidth and angular acceptance of the instrument must be small enough to provide the resolutions needed to identify narrow features in the distribution functions. The very low temperatures (down to $2000 \mathrm{~K}$ or less) expected for classical polar wind outflows can be reliably measured only with high-resolution differential energy measurements, as well as with narrow window apertures. The ability to distinguish between ion beams and conics which have been strongly folded by the magnetic mirror force also implies a high angular resolution. The necessity to satisfy these conflicting constraints on sensitivity and resolution drove the design of the instrument, and particular attention was paid to the efficiency of both ion optics and detectors. The conditions encountered at lower altitudes close to perigee differ drastically, with $\mathrm{O}^{+}$being the major ion and densities reaching several thousands of $\mathrm{cm}^{-3}$. This imposed a further constraint of large dynamical range in the conception of the detectors in order to avoid saturation or even destructive effects (cf. Sect. 2.5).

A major difficulty lies in the necessity of allowing the very low energy ions to enter the instrument, even when the satellite is exposed to sunlight and immersed in tenuous plasmas, and therefore tends to reach significant positive floating potentials. In the first part of the mission, this was achieved by means of the satellite potential control experiment RON (Torkar et al., 1998). After problems appeared in the operation of this device, the adverse effects of spacecraft potential were counteracted by applying an adjustable potential bias to the entrance surface of the experiment. The energy range up to $80 \mathrm{eV}$ is sufficient to detect $\mathrm{H}^{+}$field-aligned flows at high speeds up to about $80 \mathrm{~km} \mathrm{~s}^{-1}$ at high altitudes, as well as $\mathrm{O}^{+}$ions swept at speeds up to $20 \mathrm{~km} \mathrm{~s}^{-1}$ by the magnetospheric convection. Hyperboloid observations in the upper part of its energy range are also complementary to those performed at higher energies by the Ion experiment (Sauvaud et al., 1998).

Due to the low fluxes measured at high altitudes, there is only a limited possibility of reducing the detector integration times in order to observe possible rapid changes in distribution functions. In practice, a trade-off must therefore be found between time resolution and spacing of measurements in velocity space. The various modes of operation reflect the different compromises which have been chosen with respect to the scientific objectives (cf. Sect. 2. 6.)

\subsection{Outline}

The instrument is constituted of two multi-directional analyzers, each one encompassing several entrance 
windows (Fig. 1). The main analyzer (A16) measures ions with velocities in plane $\mathrm{P} 1$, and contains 16 directions of sight defined by an identical number of windows through the main cylindrical part of the cover which surrounds the sensor unit. These windows are positioned at $10^{\circ}$ intervals and have $\approx 13^{\circ}$ angular acceptance, so that the instrument spans a continuous $160^{\circ}$ angle in plane P1. The secondary analyzer (A10) with its view axis in plane $\mathrm{P} 2$ encompasses 10 windows positioned $15^{\circ}$ apart from each other and with $\approx 6^{\circ}$ angular acceptance, so that the total angle spanned in plane $\mathrm{P} 2$ is $145^{\circ}$ with blind sectors of $\approx 9^{\circ}$ angular extent between adjacent windows.

Figure 2 shows that plane P1 contains the spacecraft spin axis, and therefore scans the full 3D space in one spin period $\approx 120 \mathrm{~s}$. The secondary plane $\mathrm{P} 2$ makes an angle of $75^{\circ}$ with the spin axis and is inclined towards the anti-solar direction. The two analyzers exhibit complementary performances. The A16 has by far the highest geometrical factor and provides ion distribution functions in its half-plane with a time resolution of roughly a few seconds, but is able to detect ions coming from a given direction only once per spin. When combined with the A16, the A10 offers partial access to the 3D features of ion distributions with a much better time resolution (typically a few seconds). Although its performances in terms of geometrical factor are much below those of the A16, it enables one to follow the temporal variations of ion flows over approximately half a spin period when the ion bulk velocity is adequately oriented.

Hyperboloid ion optics consists of three main parts, as illustrated in Fig. 3:

1. the electrostatic optics comprising the entrance sections and the spherical analyzers used to select the direction of sight and the energy interval of the

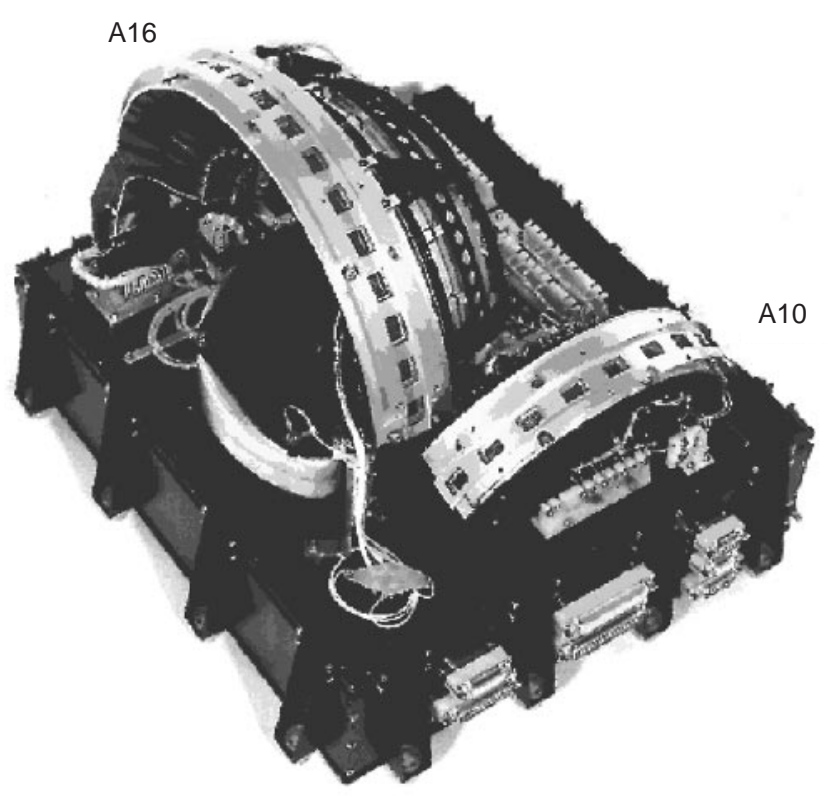

Fig. 1. Photograph of Hyperboloid without its external cover

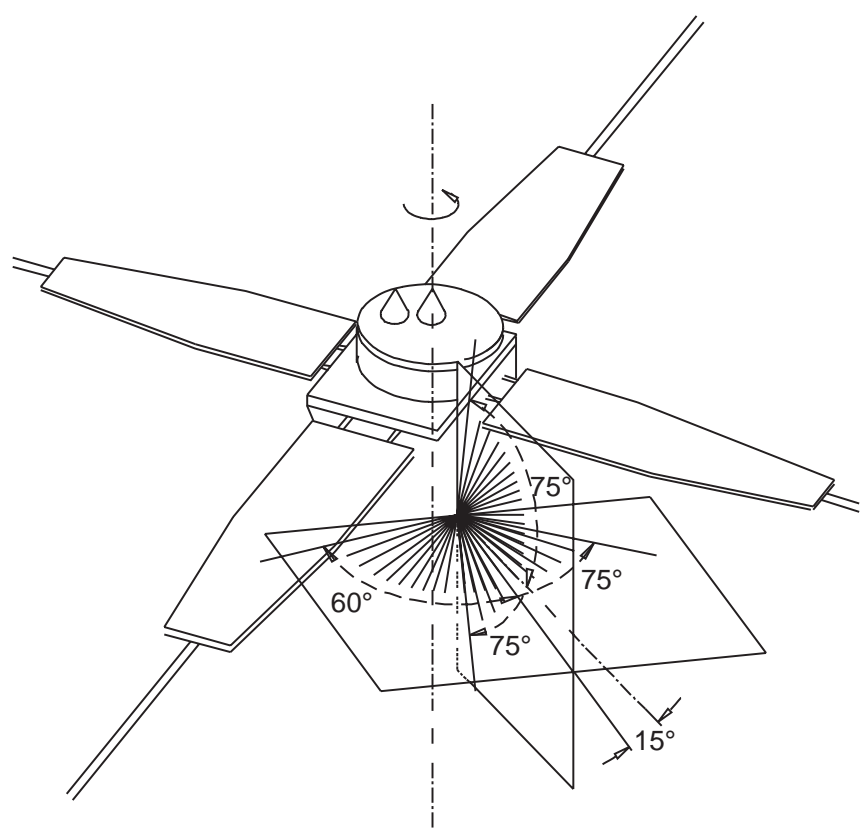

Fig. 2. Geometry of the two analyzers relative to the spacecraft

measurements, respectively, and the toroidal concentrator whose function is to optimize the conditions of entry inside the magnetic analyzer;

2. the magnetic analyzer which separates the various ion species;

3. the micro-channel plates (MCP) detectors.

\subsection{Electrostatic optics}

The most external grid of each entrance window is biased at potential $V_{\text {bias }}$ relative to the satellite, together with the cylindrical part of the external cover of the experiment. As explained in Sect. 2.1, this is intended to compensate spacecraft charging effects. All the other voltages of the electrostatic optics are referenced to $V_{\text {bias, }}$ except for the very high voltages near the exit of the concentrator for which this precaution is unimportant. The second grid of the entrance section can be switched from $V_{\text {bias }}$ to $+200 \mathrm{~V}$ independently for each window, so that any combination of windows can be opened simultaneously. This enables the adaptation of the angular resolution and sensitivity of the instrument to the various scientific objectives.

Each window of the A16 is followed by an individual spherical energy analyzer composed of two electrodes whose voltages are controlled in order to select ions around a given energy $E$, and to deflect them by $90^{\circ}$ toward the entrance of the toroidal concentrator. A retarding or accelerating potential $V_{r}$ can be applied to the entrance and exit grids of the energy analyzer, the potentials applied to the electrodes being adjusted accordingly. As a result, the energy bandwidth of the analyzer is multiplied from its nominal value by a factor $K=1-V_{r} / E$, and the angular acceptance is changed 


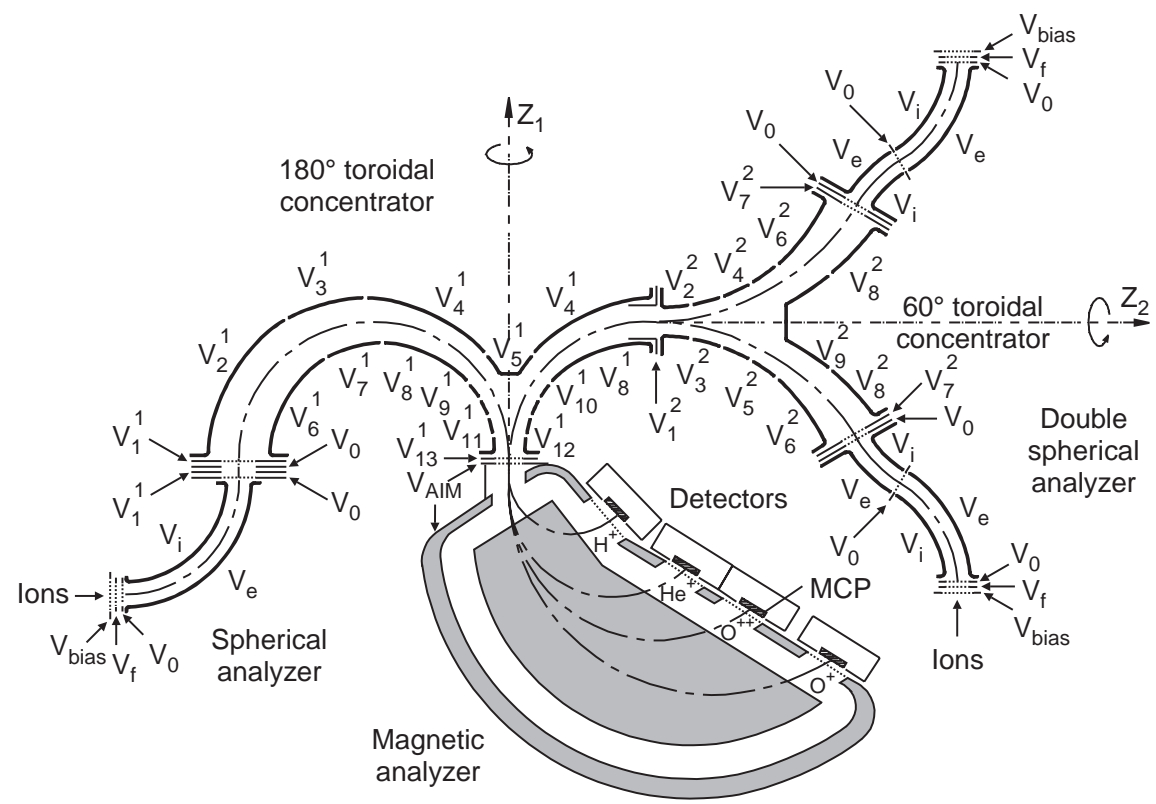

Fig. 3. Schematic cross section of the ion optics in the plane of the magnetic analyzer. The electrostatic optics on the left of the figure is representative of the first window of the main analyzer (A16), the optics of the other windows of the A16 are obtained by successive rotations of $10^{\circ}$ around axis $Z_{1}$. Similarly, the optics figured on the right are relevant for windows 1 and 10 of the secondary analyzer (A10), optics for the other windows being deduced by $15^{\circ}$ rotations around axis $Z_{2}$ approximately in the same ratio. Following the energy analyzer section is the main toroid concentrator made up of an assembly of independently polarized electrodes whose voltages have been optimized so that, irrespective of their entrance window, ions enter the magnetic analyzer as a nearly cylindrical beam with small angular divergence.

Each window of the A10 is followed by two consecutive spherical electrostatic analyzers with opposite curvature radii, deflection angles of $60^{\circ}$ and $30^{\circ}$, respectively, and again with adaptable retarding/accelerating potential $V_{r}$. This particular geometry was imposed by the overall mechanical constraints of the instrument but has the advantage that the aberrations of the first analyzer are partly counterbalanced by the second one. Ions exiting from these energy analyzers enter a secondary toroidal concentrator which directs them to the main concentrator, where they travel in the same way as ions from the A16.

\subsection{Magnetic analyzer}

Following the exit of the main toroid, ions enter the magnetic analyzer. With the nominal magnet voltage, the four routinely measured ions $\mathrm{H}^{+}, \mathrm{He}^{+}, \mathrm{O}^{++}$, and $\mathrm{O}^{+}$are focused on their respective detector. Sweeping this voltage enables to detect minor ions such as $\mathrm{N}^{+}$or molecular ions.

The geometry of the magnet analyzer is derived from the Mattau-Herzog geometry but has been optimized through ray-tracing calculations taking into account the actual geometry of the ion beam at its entrance. In particular, an improved focusing is obtained by using an entrance plane non normal to the mid-axis $Z_{1}$ (Fig. 3) and by choosing an angle for the exit face of the magnet which is not the same for $\mathrm{H}^{+}$as for all other ions. This effort in design enabled a significant reduction of ion losses by improving the acceptance of the magnetic analyzer for trajectories inclined with respect to its midplane of symmetry.

\subsection{Detectors}

The four detectors use curved micro channel plates (MCP) fabricated by Photonix-Mullard Company with $\mathrm{C}$-shaped channels $25 \mu$ in diameter and $2 \mathrm{~mm}$ long. The average gain is $1.5 \cdot 10^{6}$ at $1600 \mathrm{~V}$ and the resolution on the order of $80 \%$. Each MCP is connected to its own high-voltage (HV) power supply.

The detectors may be operated either in a pulse counting mode for ion fluxes lower than $1.5 \cdot 10^{5} \mathrm{c} / \mathrm{s}$, or in an analog mode with charge integration for larger fluxes. In order to maintain the output current above the noise level, but also below $2 \%$ of the polarization current (thus keeping the MCP operation in the linear range), the mode of measurement and, when in the analog mode the HV applied to the MCPs, are automatically controlled on board by the microprocessor. The gain of the MCPs is thus permanently adapted to the registered ion fluxes. The resulting total dynamical range is larger than $10^{11}$, more than convenient for the maximum of $10^{10} \mathrm{c} / \mathrm{s}$ foreseen at perigee for $\mathrm{O}^{+}$.

\subsection{Modes of operation}

Several modes types have been defined to operate the instrument. Beside technical modes intended to check the health of the electronics and dump the memory, and diagnostic modes used to monitor any evolution in the physical response of the instrument optics and detectors, scientific modes consist in repetitive measurements where both energy and view direction are swept in sequence during several spacecraft spin periods. 
Each scientific mode is defined by:

1. the set of measured ion species $\left(N_{\text {ion }}=\right.$ between 2 and 4 species taken in the $\mathrm{H}^{+}, \mathrm{He}^{+}, \mathrm{O}^{++}$, and $\mathrm{O}^{+}$ group);

2. the energy sweep, consisting of a geometrical progression characterized by its initial energy $E_{0}$, progression factor $\rho$ and number of energies $N_{\text {energy }}$. The energy resolution $\Delta E / E$ may be chosen either constant (fixed $K$ ), or energy dependent (fixed $V_{r}$ ), as defined in Sect. 2.3;

3 . the window sweep consisting of one or two progressions including either A16 or A10 windows. Each progression is characterized by its initial window $W_{0}$, number of window groups $N_{\text {group }}$, and number of windows per group $N_{\text {window. }}$ The window shift between two consecutive groups $N_{\text {step }}$ can be chosen different from $N_{\text {window }}$ so that consecutive groups can overlap;

4. the double-sweep structure, with internal loop either on energies (DE mode) or on window group (DA modes).

Independently to the scientific mode, the telemetry systems collect data into so-called "pages", each of which contains 72 individual measurements. A complete sweep over energies and window groups is therefore performed in $N_{\text {page }}=\left(N_{\text {ion }} \times N_{\text {energy }} \times N_{\text {group }}\right) / 72$ pages, i.e., $N_{\text {page }} / T M$ seconds, where $T M$ is the instrument telemetry rate expressed in pages per second. This time is the temporal resolution for obtaining distribution functions in the half-plane of one or two of the analyzers. It is also linked to the angular resolution obtained in the spin plane. The choice of the mode attributes therefore implies a trade-off between energy, angular, and temporal resolution.

The commanding of instrument operations is organized through a hierarchy of structures. The so-called "enchainments" include one or several modes, with a prescribed duration in units of the spacecraft spin period. Enchainments are further assembled into "sequences" chosen by operating telecommands which also define the potential bias $V_{\text {bias }}$ and the telemetry rate $T M$. For safety reasons, the information defining all modes, enchainments, and sequences were loaded in LU tables in two redundant EEPROM memories. Table 1 summarizes the characteristics of the most commonly used sequences, which favor either energy resolution (large $N_{\text {energy }}$ and small $\rho$ ), angular resolution in the A16 plane (small $N_{\text {window }}$ ), or temporal resolution, equivalent to angular resolution in the spin plane (small $N_{\text {page }} / T M$ ).

\section{Data reduction}

For a given ion species, measurements performed at times $t_{k}$ during a set of successive swaeps over energy steps $E_{i}$ and window groups $W G_{j}$ form a $3 \mathrm{D}$ array of ion counts $N_{i j k}$ The measured distribution function $F_{i j k}$ and differential fluxes $J_{i j k}$ can be related to counts by:

$N_{i j k}=\tau \times G_{i j} \times F_{i j k} \times E_{m i}^{2} \times 2 / M^{2}$,

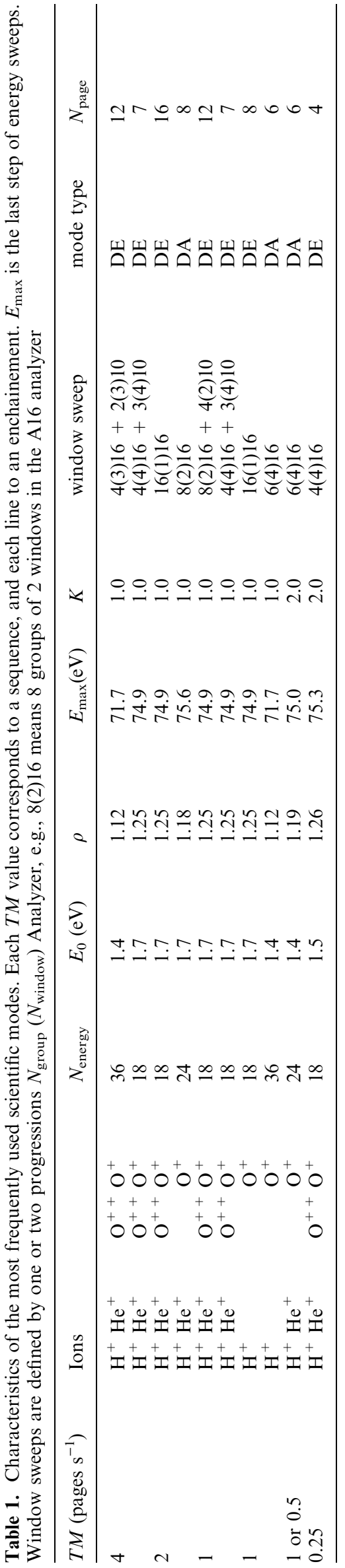


$J_{i j k}=F_{i j k} \times E_{m i} \times 2 / M^{2}$,

where $\tau$ is the integration time (which varies between 4 and $200 \mathrm{~ms}$, depending on the number of measured ion species and on the telemetry rate), $G_{i j}$ the geometrical factor, and $M$ the ion mass. $E_{m i}=Q \times E_{i}$ is the measured energy of ions of charge $Q$ for energy step $E_{i}$.

The geometrical factors may be written as:

$G_{i j}=T R_{i j} \times K^{2} \times \Delta E / E \times A_{j} \times \delta \Omega_{i j}$,

where $K$ is the resolution factor (cf. Sect. 2.3), $\Delta E / E$ the nominal energy resolution of the spherical analyzer $(0.23$ and 0.09 for A16 and A10, respectively), and $A_{j}$ the surface of individual windows $\left(1.4 \mathrm{~cm}^{2}\right.$ for $\mathrm{A} 16$ and $0.84 \mathrm{~cm}^{2}$ for A10 windows). $\delta \Omega_{i j}$ represents the sum of the nominal acceptance solid angles of windows in group $G W_{j} \quad\left(13^{\circ} \times 12.5^{\circ}\right.$ and $6^{\circ} \times 3^{\circ}$, corresponding to $3.9 \cdot 10^{-2}$ and $4.3 \cdot 10^{-3}$ sr for each window of the A16 and A10, respectively), and $T R_{i j}$ the transparencies which depend on ion species, energy step, and window group.

Each value $F_{i j k}$ is a discrete measurement of the ion distribution function associated to the corresponding energy and direction at the entrance of the instrument. By taking into account the effect of the spacecraft sheath and applying Liouville's theorem, one can then deduce a set of discrete values of the distribution function in the plasma, but such complex calculations are not possible at this early stage of data reduction. In view of the generally large Debye length compared to the size of the instrument, we have considered as a first approximation the sheath to be spherically symmetrical over the half space of viewing directions, which implies that ion directions of arrival are unaffected. Moreover, ion energy in the plasma is given by:

$E_{p i}=E_{m i}+Q \times V_{\exp }=Q \times\left(E_{i}+V_{\text {sat }}+V_{\text {bias }}\right)$,

where $E_{i}$ is the energy step and $V_{\exp }$ and $V_{\text {sat }}$ the experiment and satellite potentials, respectively.

In principle, $V_{\text {sat }}$ can be determined directly from DC electric field measurements from the IESP experiment (Perraut et al., 1998). However, such measurements might be difficult to interpret at high altitude when the Debye length becomes larger than the antenna length. Therefore, we have tried to develop a fast method to determine $V_{\text {sat }}$ relying on Hyperboloid data only. A set of $V_{\text {sat }}$ values is used to calculate the undisturbed ion energies from Eq. (4), from which the moments (density, velocity vector, parallel and perpendicular temperatures) of the undisturbed distribution function are deduced. Each $V_{\text {sat }}$ and associated moments are then introduced as input parameters of a program simulating the working of the experiment. This program is presently based on a bi-Maxwellian model of distribution functions, which is generally a reasonable approximation. The best fit between simulated and experimental fluxes provides the estimation of $V_{\text {sat }}$ and the corresponding moments. As shown in Sect. 4, this simple method produces results which are satisfactory for preliminary data analysis; it will be improved in the future by taking into account more accurate sheath effects.

\section{Preliminary results}

After more than one year of activity, the Interball Auroral spacecraft has covered all MLT sectors at subauroral, auroral, and polar-cap latitudes in the northern hemisphere, and at altitudes between about 10000 and $20000 \mathrm{~km}$. The data collected by Hyperboloid during this period reveal a wide variety of phenomena which we illustrate by three typical examples.

\subsection{Ion outflow and wave-induced heating in auroral regions}

Figure 4 displays a set of wave and particle measurements performed between 21:00 and 22:00 UT on 2 December 1996 over the northern near-midnight auroral zone and polar cap, and at altitudes between about 16000 and $19000 \mathrm{~km}$.

First, a region of ion heating is crossed from about 21:00 and 21:24 UT, characterized by $\mathrm{H}^{+}$fluxes almost uniform over nearly the entire energy range. These fluxes also exhibit little dependence on spacecraft spin (2 min), which implies almost isotropic $\mathrm{H}^{+}$distributions typical of strong heating. Due to the high altitude, and probably also to the winter and solar minimum conditions, $\mathrm{O}^{+}$fluxes are much smaller, and even close to noise level at low energies. However, they extend over the full instrument energy range, which shows that $\mathrm{O}^{+}$ as well as $\mathrm{H}^{+}$ions are heated. Due to their higher mass, $\mathrm{O}^{+}$ions tend to have smaller thermal velocity, which explains why their fluxes are more collimated in azimuth angles and therefore exhibit noticeable temporal variations over a spin period.

This period of ion heating is associated with strong low-frequency turbulence, as evidenced by the second top panel of Fig. 4 representing the low-frequency wave magnetic component measured in the direction parallel to the spacecraft spin axis by the IESP experiment [courtesy of S. Perraut and M. Mogilevsky, see also Perraut et al. (1998)]. Significant power is observed around the oxygen gyrofrequency $f_{c+} \approx 0.5$ to $0.9 \mathrm{~Hz}$, up to the hydrogen gyrofrequency $f_{c \mathrm{H}+} \approx 9$ to $14 \mathrm{~Hz}$, and probably at higher frequencies, where unfortunately no data are available. Other electric and magnetic components (not shown) are characterized by similar behavior. During the same time-interval, electron fluxes measured by the ion experiment between about $10 \mathrm{eV}$ and $20 \mathrm{keV}$ [courtesy of J.-A. Sauvaud, see also Sauvaud et al. (1998)] show keV electrons, probably of plasma sheet origin, accompanied by impulsive fluxes at lower energies down to about $30 \mathrm{eV}$ (the intensification at $20 \mathrm{eV}$ is due to photoelectrons returning toward the positively charged satellite). It is tempting to interpret the waves as Alfvèn-like electromagnetic turbulence destabilized by the observed electron distributions. Indeed, these waves are reminiscent of those observed at lower altitudes by the Viking satellite (Gustafsson et al., 1990). In the latter reference, it is shown that 


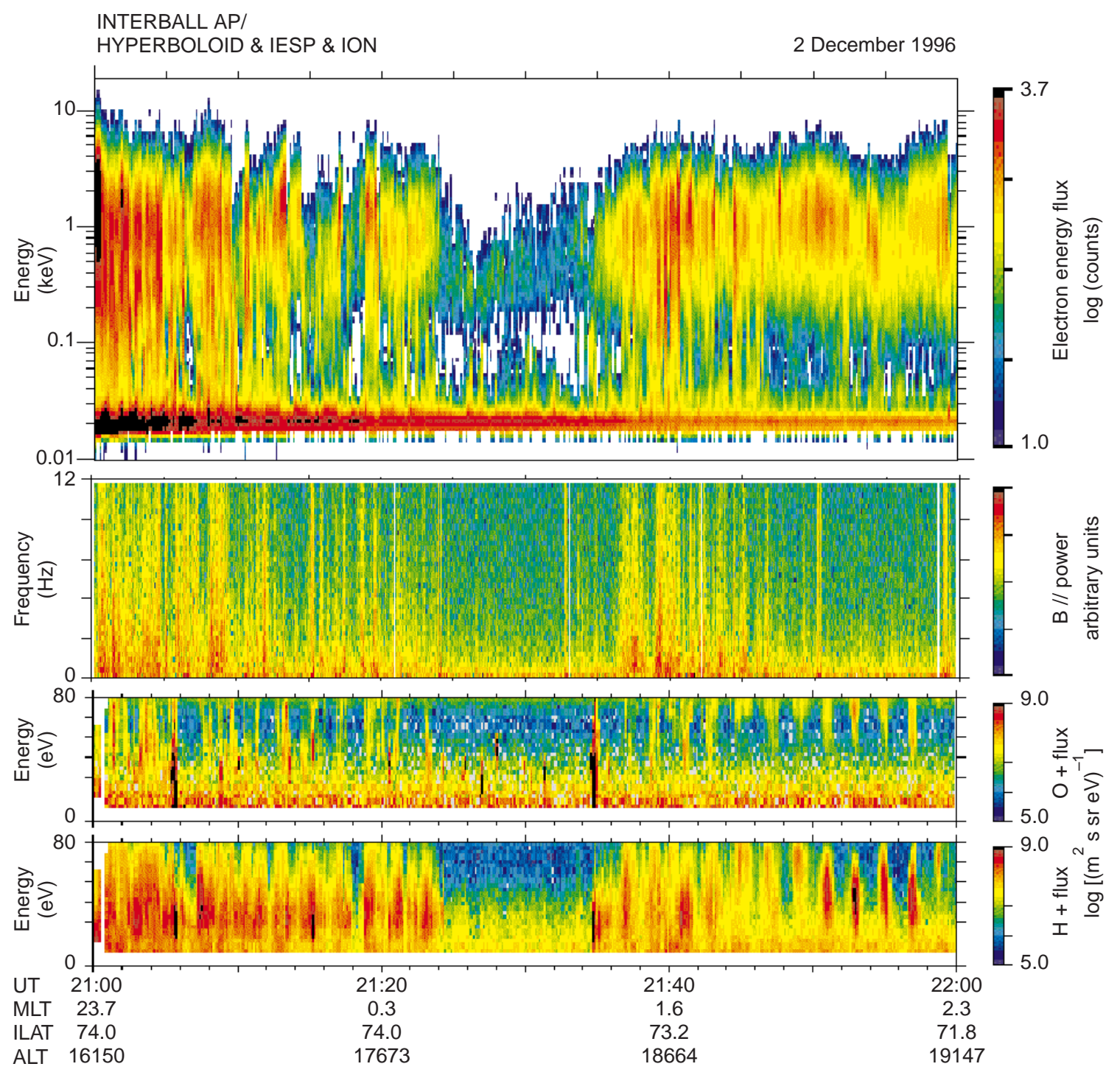

Fig. 4. Wave and particle measurements obtained on 2 December 1996 between 21:00 and 22:00 UT, in the midnight MLT sector of auroral zone and polar cap. From top to bottom, $10 \mathrm{eV}-20 \mathrm{keV}$

electron distributions with several populations including a field-aligned beam are able to destabilize a shear Alfvèn mode. Although the magnetic field line configuration during the period analyzed here does not enable to estimate electron fluxes outside of the 50 to $130^{\circ}$ pitch angle range, the electrons detected below $1 \mathrm{keV}$ appear as a plausible energy source for the waves (Temerin and Lysak, 1984).

Figure 5 displays time-window spectrograms in different energy ranges during one spin period between 21:02 and 21:04 UT characterized by an intense electromagnetic turbulence. Above $30 \mathrm{eV}, \mathrm{H}^{+}$and $\mathrm{O}^{+}$maximal fluxes fall almost systematically in the 90 to $130^{\circ}$ pitch angle range, suggesting that ions are heated perpendicular to the magnetic field below the satellite, between about 13000 and $16000 \mathrm{~km}$. H ${ }^{+}$fluxes at lower energies gradually shift toward the upward field-aligned direction (the corresponding $\mathrm{O}^{+}$fluxes are close to noise level and are not plotted). Such simultaneously observed electron counts from Ion, magnetic component parallel to the spin axis of waves below $12 \mathrm{~Hz}$ from IESP, and $0-80 \mathrm{eV} \mathrm{O}^{+}$and $\mathrm{H}^{+}$ fluxes averaged over Hyperboloid half-planar field of view

$\mathrm{H}^{+}$and $\mathrm{O}^{+}$conics are different from those observed at similar altitudes but higher energies by DE-1 (Klumpar et al., 1984), which were shown to result from cumulative ion cyclotron resonance (ICR) heating over almost the entire field line (Crew et al., 1990). In the event analyzed here, ICR with the low-frequency electromagnetic turbulence detected by IESP is a plausible scenario for explaining the observed $\mathrm{H}^{+}$and $\mathrm{O}^{+}$ion heating, even if the contribution of higher frequency, e.g., lower hybrid, waves cannot be ruled out (André et al., 1994). This suggests that ICR may on some occasions occur over a limited altitude range, thus leading to conics of moderate energies. Another theoretical question raised by the present observations is the possibility of obtaining simultaneous heating of both $\mathrm{H}^{+}$and $\mathrm{O}^{+}$by ICR, while classical theories describe heating of minor species only (Le Quéau et al., 1993).

On Fig. 4, the period of ion heating ceases at about 21:24 UT, simultaneously with the disappearance of 

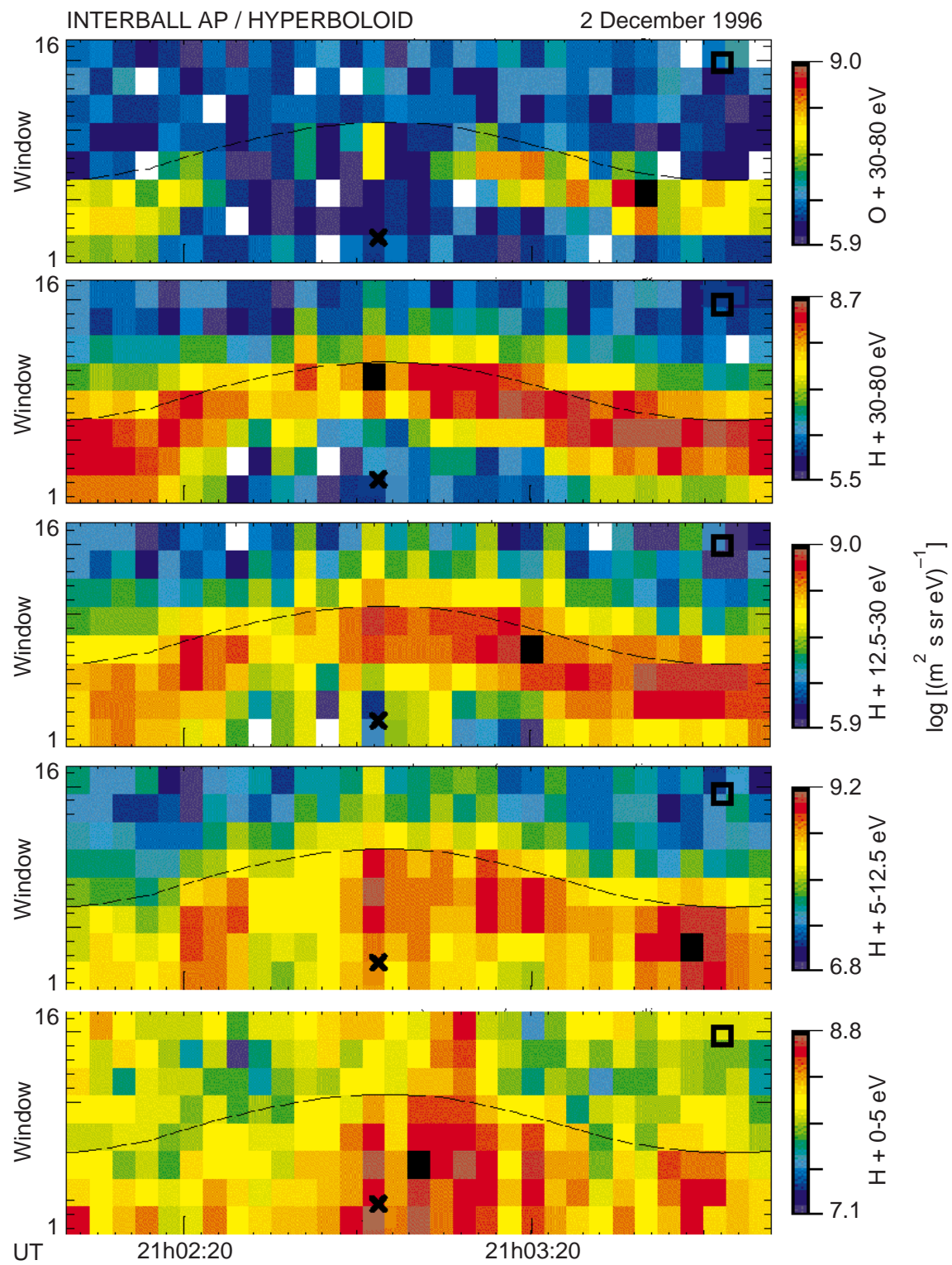

Fig. 5. 3D distribution function of $\mathrm{H}^{+}$and $\mathrm{O}^{+}$ions on 2 December 1996 between 21:02 and 21:04 UT. Each spectrogram displays the angular variation of fluxes in a given energy range. The black crosses and squares indicate ions moving up and down along the magnetic field lines, respectively, while the full line corresponds to $90^{\circ}$ pitch angles low-frequency waves, and with a significant drop in electron fluxes and average energies. In the time-interval between 21:24 UT and 21:35 UT, probably corresponding to the polar cap, narrow $\mathrm{O}^{+}$ion distributions are observed, while $\mathrm{H}^{+}$fluxes remain below the noise level. It may be noted that the higher background fluxes observed at lower energies for both $\mathrm{H}^{+}$and $\mathrm{O}^{+}$ions during this time-interval correspond to small counts close to the noise level, which induce artificially enhanced fluxes due to the lower instrument transparencies at lower energies (cf. Eqs. 1-3). This feature is also visible on Figs. 7, 8, and 9 below. Close to 21:28 UT on Fig. 4, we observe a narrow $\mathrm{O}^{+}$distribution at energies $\approx 10-20 \mathrm{eV}$. For such energies spacecraft potential can in first approximation be neglected, and moment calculation provides $n_{0+} \approx 1 \mathrm{~cm}^{-3}$, upward bulk velocity $v_{/ /} \approx-6 \mathrm{~km} \mathrm{~s}^{-1}$, and horizontal velocity $v_{\perp} \approx 10 \mathrm{~km} \mathrm{~s}^{-1}$. Temperature is difficult to estimate due to the very collimated fluxes. Polar outflow theories taking into account the effect of centrifugal acceleration due to ion convection across curved magnetic field lines (Cladis, 1986) indeed foresee that $\mathrm{O}^{+}$can remain the major ion at such high altitudes. Our $v_{\perp} \approx 10 \mathrm{~km} \mathrm{~s}^{-1}$ can mainly be attributed to magnetospheric convection and corresponds to a convection electric field of about $70 \mathrm{mV} \mathrm{m}{ }^{-1}$ when mapped at $200 \mathrm{~km}$ altitude. The corresponding $\mathrm{O}^{+}$density and flow velocity found by Ho et al. (1997) at $3 \mathrm{R}_{\mathrm{E}}$ altitude are in good qualitative agreement with our experimental values (see their Fig. 2). Other $\mathrm{O}^{+}$distributions registered around $5 \mathrm{eV}$ might correspond to slower convection but need to be corrected from spacecraft potential before being further analyzed.

At about 21:35 UT the spacecraft enters a new region of local ion heating, whose characteristics are initially very similar to those described in the foregoing. Figure 4 reveals however a gradual change in both $\mathrm{H}^{+}$and $\mathrm{O}^{+}$ fluxes which, starting at about 21:42 UT, show clearer spin modulations. This transition appears more clearly on Fig. 6, which shows the evolution of the $\mathrm{H}^{+}$ion 

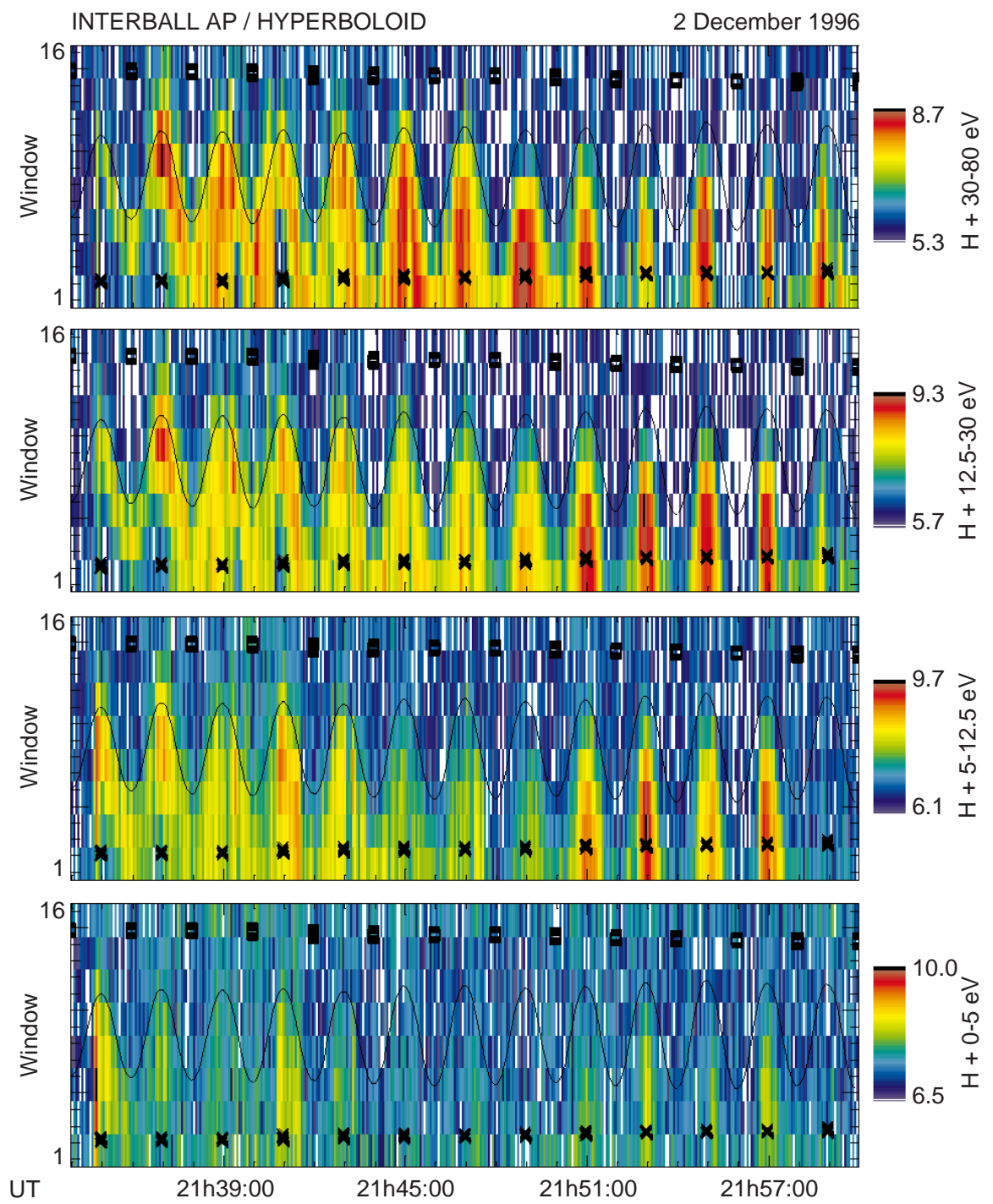

Fig. 6. Evolution of the $\mathrm{H}^{+}$distribution function on 2 December 1996 between 21:34 and 22:00 UT, in the same format as Fig. 5 distribution between 21:34 and 22:00 UT. Initially, intense fluxes are observed all along the spin periods close to $90^{\circ}$ pitch angles. At later times, fluxes exhibit a clear spin period modulation and are maximal at pitch angles close to the upward field-aligned direction. Notice that this evolution is accompanied by a decrease in the low-frequency wave spectral densities and in the 20 to $300-\mathrm{eV}$ electron fluxes (Fig. 4), in agreement with the scenario previously proposed for explaining ion heating. The distribution functions measured from about 21:44 UT are characteristic of the ion outflow processes which are frequently observed by Hyperboloid at auroral latitudes. Using the method presented in Sect. 3 , we estimated the spacecraft potential and deduced the moments of the $\mathrm{H}^{+}$distribution between 21:52 and 21:54 UT. We found $V_{\text {sat }} \approx+7 \mathrm{~V}$, and corresponding values for density $n \approx 0.8 \mathrm{~cm}^{-3}$, upward bulk velocity $v_{/ /} \approx-42 \mathrm{~km} \mathrm{~s}^{-1}$, horizontal velocity $v_{\perp} \approx 13 \mathrm{~km} \mathrm{~s}^{-1}$, parallel temperature $T_{/ /} \approx 1.5 \mathrm{eV}$, and perpendicular temperature $T_{\perp} \approx 2 \mathrm{eV}$. At the same moment, $n \approx 0.7 \mathrm{~cm}^{-3}$ and $v_{/ /} \approx-18 \mathrm{~km} \mathrm{~s}^{-1}$ for $\mathrm{O}^{+}$. Considering the balance between currents generated by satellite photo-electrons and by ambient thermal electrons of density $n_{e} \approx n_{\mathrm{H}+}+\mathrm{n}_{0+} \approx 1.5 \mathrm{~cm}^{-3}$ and temperature $T_{e} \approx 2 \mathrm{eV}$, leads to an estimate $V_{\text {sat }} \approx+6 \mathrm{~V}$, which is a good confirmation of the validity of our method for calculating moments corrected from satellite charging effects. The same qualitative results are obtained all throughout the 21:44 to 22:00 UT period, i.e., the $\mathrm{H}^{+}$ and $\mathrm{O}^{+}$outflowing ions exhibit different bulk velocities and different mean energies. Such a mass-dependent acceleration process might result from a bimodal acceleration involving (1) magnetic moment pumping due to fluctuating electric fields (e.g., associated to waves similar to those observed between 21:00 and 21:24 UT but acting at lower altitudes), and (2) field-aligned acceleration by quasi-static electric fields (Lundin and Hulqvist, 1989).

\subsection{Thermal ion signatures of large-scale boundaries in the nightside magnetosphere}

Thermal and suprathermal plasma emanating from the ionosphere is affected by magnetospheric plasma 
processes at high altitudes such as convection, fieldaligned currents, heating, and acceleration. It is therefore interesting to look at the changes of the thermal plasma parameters at boundaries between the largescale magnetospheric plasma domains.

Methods to locate these boundaries in the night sector from measurements of precipitating particles on low-altitude satellites were developed during the last two decades and now seem well established (Feldstein and Galperin, 1985; Galperin and Feldstein, 1991, 1996; Newell et al., 1996). According to the review of Feldstein and Galperin (1985), the soft electron precipitation boundary (SEB) practically coincides with the equatorial large-scale convection boundary. Other hot plasma regimes - above the diffuse auroral zone (i.e., within the trapping boundary of high-energy particles), above the oval of discrete auroral forms (i.e., within the so-called auroral plasma cavity), and further poleward - can also be delineated during stationary conditions. The characteristics of thermal ion populations at mid-altitudes in the plasmasphere and above auroral oval and polar cap were extensively analyzed from data on DE-1 (Horwitz et al., 1986; Giles et al., 1994), and Akebono (Abe et al., 1993; Watanabe et al., 1992). These experiments have demonstrated the predominance of polar wind flows, and revealed regions of counterstreaming flows as well as many other features. Horwitz et al. (1986) analyzed thermal plasma distributions with respect to the hot plasma boundaries in the inner magnetosphere from electron data on DE-1 and 2. They concluded from a statistical analysis that in the nightside the SEB practically coincides with the plasmapause. However, they did not analyze the thermal plasma signatures associated to the auroral oval of discrete forms and polar-cap boundaries. In this paper, from a data set corresponding to a quieting period after a storm, we present a preliminary investigation of the thermal plasma characteristics at, and between, the boundaries of the main magnetospheric plasma domains.

We chose the nightside parts of a series of similar orbits on 12-13 January 1997, when the magnetosphere was recovering from a period of strong magnetic activity induced on 10-11 January by a very dense "solar plasma cloud" which engulfed the circumterrestrial space. This was a period of outer plasmasphere refilling with some weak activity $\left(2-\leq K_{p} \leq 3-\right)$. Consider the nightside part of the inbound orbit 565 in the reverse chronological order, from the nightside plasmasphere poleward across the diffuse auroral zone, oval and polar cap (Fig. 7). From the simultaneous data from Ion (courtesy of J.-A. Sauvaud), Interball AP crossed the equatorial border of the diffuse auroral zone, defined as the region of increasing plasma sheet electron energy with latitude, at 06:30 UT (MLT $\approx 0.6$, invariant latitude $\approx 64.3^{\circ}$ ). The polar edge of the diffuse zone, i.e., the equatorial border of the oval, was probably crossed in the interval 06:12-06:14 UT corresponding to the maximum observed electron energies. Then the satellite entered the plasma sheet type plasma, with a gradually decreasing average energy and some structuring of electron fluxes till its polar edge at 05:35 UT. There, an enhancement of energetic electron fluxes and average energies, accompanied by a velocity dispersed ion structure of type 2 (VDIS-2) (Zelenyi et al., 1990), was observed at about 05:40 UT, followed by a decrease in the average electron energy. This last feature may be identified as a narrow polar diffuse zone at the polar edge of the oval.

Figure 7 shows that significant changes in the thermal plasma characteristics occur at or close to these hot plasma boundaries. A very similar latitudinal structure of the particle data was observed on the other orbits of

INTERBALL AP / HYPERBOLOID \& ION

12 January 1997

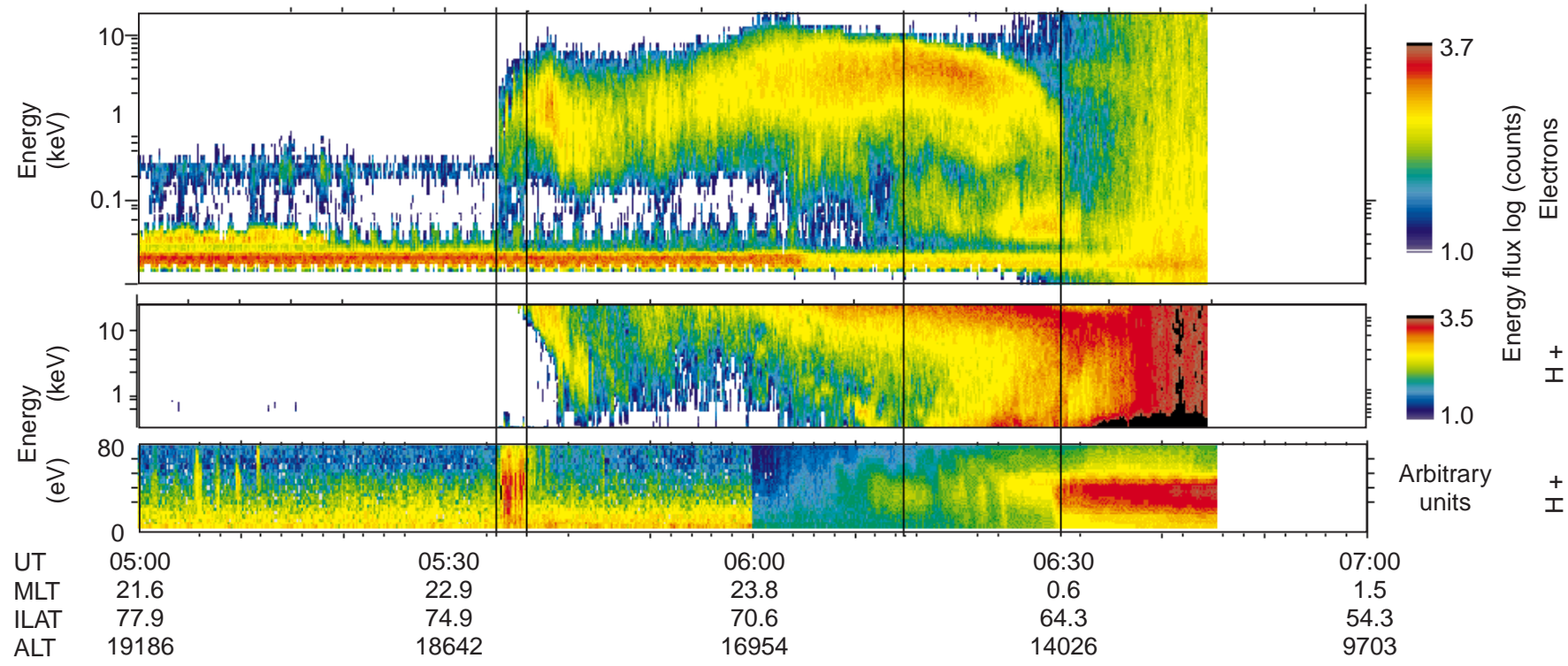

Fig. 7. From top to bottom, energy-time spectrograms for highenergy electrons and $\mathrm{H}^{+}$ions from Ion, and for $0-80 \mathrm{eV} \mathrm{H}^{+}$ions from Hyperboloid between 05:00 and 07:00 UT on 12 January 1997.
Note that two different color scales have been used in the bottom panel in order to enlighten both low fluxes before 06:00 UT and large fluxes after this time 
this period. We now summarize the thermal and suprathermal plasma characteristics within the various magnetospheric domains.

Outer plasmasphere and plasmapause. The region of space within the stationary, or quasi-stationary, convection boundary, is evidently the outer plasmasphere, and the boundary is the plasmapause. Indeed, a sharp drop in the thermal plasma density from more than $100 \mathrm{~cm}^{-3}$ to values lower by one to two orders of magnitude is evident at, or close to, the SEB. Densities recorded on successive orbits before orbit 565 were much lower $\left(\approx 0\right.$ and $\left.8 \mathrm{~cm}^{-3}\right)$, suggesting that refilling was ongoing in the outer plasmasphere region. We defer the analysis of the refilling rates to a further publication.

Diffuse auroral zone. The plasma density outside the plasmapause continues to decrease with latitude down to very low values. In this region the positive potential of the satellite increases simultaneously, so that a more refined data analysis is needed to deduce a reliable latitudinal density profile. However, since the average ion energy is fairly constant, it is anticipated that the observed relative variations of ion fluxes are correct. For orbits 565 and 569 , a narrow region of counterstreaming $\mathrm{H}^{+}$and $\mathrm{He}^{+}$ ions exists near the plasmapause at energies above $\approx 15 \mathrm{eV}$.

Plasma cavity above the auroral oval. Very low, but measurable thermal plasma fluxes are seen above the auroral plasma forms: this is the well-known auroral plasma cavity (Calvert. 1981) where the hot plasma sheet component generally dominates the total plasma density. Sometimes filaments of denser, primarily outflowing thermal plasma are crossed, which might play a role in wave propagation and wave-particle interactions.

Polar edge of the oval. A new feature is discovered in the thermal plasma, coincident with the low-energy electron precipitation observed at the polar edge of the auroral oval of discrete arcs, and with the extrapolation to infinite velocity of the associated VDIS. It is seen on all orbits considered here, and appears as a density peak of thermal and suprathermal $\mathrm{H}^{+}$and $\mathrm{He}^{+}$ions with a wide spectrum from the lowest energies till the upper end of Hyperboloid energy range. The $\mathrm{H}^{+}$densities in the peak reach $0.15 \mathrm{~cm}^{-3}$ on orbits 565 and 569 , and $0.05 \mathrm{~cm}^{-3}$ on orbit 571 . The energy spectrum of these suprathermal ions can extend to a few hundreds of eVs (middle panel of Fig. 7). The analysis of ion velocities shows predominant upward directed fluxes, but also a sizable fraction of downgoing ions. This suggests the combined action of ionospheric ion heating at low altitudes due to electron precipitation, and of ion heating or scattering all along the field lines probably due to wave-particle interactions.

Polar cap. The thermal plasma distributions in the polar-cap region adjacent to the auroral oval are similar to those in the auroral cavity with extremely low densities practically at or below the instrument threshold $\approx 10^{-3} \mathrm{~cm}^{-3}$. A distinct change in plasma characteristics is identified at $77^{\circ}$ invariant latitude with the appearance of $\mathrm{H}^{+}$and $\mathrm{He}^{+}$outflowing ions with densities $\approx 10^{-2} \mathrm{~cm}^{-3}$ and velocities $\approx 20 \mathrm{~km} \mathrm{~s}^{-1}$ typical of heated polar wind conditions. Abrupt changes in the properties of these outflows can be seen between consecutive spins. Such changes are frequent in Hyperboloid observations and might be the consequence of small-scale structures in the ionosphere.

\subsection{Low-energy ion dynamics in the cusp/cleft region}

Five months after its launch, Interball AP began to explore the cusp/cleft region characterized by precipitation of plasma from the magnetosheath (Heikkila and Winningham, 1971; Reiff et al., 1977; Burch et al., 1982; Menietti and Burch, 1988; Newell and Meng, 1988; Kremser and Lundin, 1990). Another common feature is the observation of low-energy (below $\approx 100 \mathrm{eV}$ ) ionospheric plasma outflows (Lockwood et al., 1985; Horwitz and Lockwood, 1985; Pollock et al., 1990; Giles et al., 1994). Two representative crossings of the cusp/ cleft region are examined here.

Figure 8 shows particle data acquired by the Hyperboloid and Ion (courtesy of J.-A. Sauvaud) experiments on 26 February 1997, as the spacecraft crossed the cusp, moving poleward and toward the post-noon dusk sector. Interball AP crossed the equatorward boundary of the cusp at 00:18 UT (12.7 MLT and $75^{\circ}$ invariant latitude), as indicated by the magnetosheath electrons encountered below $\approx 200 \mathrm{eV}$ until about 00:40 UT (77. $7^{\circ}$ invariant latitude). Associated to the 120-s satellite spin period, V-shaped ion signatures characteristic of the direct injection of magnetosheath particles in the cusp appear in the $\mathrm{H}^{+}$data around $2 \mathrm{keV}$ at 00:20 UT ( $\approx 12.8 \mathrm{MLT}$ and $75.2^{\circ}$ invariant latitude), slightly poleward of the low-latitude boundary of the electron precipitation region. This $\mathrm{V}$-shaped pattern is explained by transit-time effects, assuming an injection source of narrow latitude extent located in the exterior cusp. The thickness of each V structure depends on the source extent. The gradual energy decrease observed as the satellite moves toward higher latitude is due to antisunward convection (Burch et al., 1982). The V-shaped $\mathrm{H}^{+}$ features begin to be seen by Hyperboloid from about 00:36 UT and show a remarkable continuity with those observed at higher energies. Another striking feature is the inversion of the $\mathrm{H}^{+}$ion $\mathrm{V}$ structure observed around 00:42 UT, apex of the V's appearing at higher energies than the wings after this time: this is explained by the fact that progressively, the downgoing ions do not reach the satellite any more and only upward moving ions which have mirrored below the satellite are observed (Burch et al., 1982). Injected $\mathrm{H}^{+}$ions are seen by Hyperboloid down to about $5 \mathrm{eV}$. Even taking into account possible spaceraft charging effects, this is well below typical magnetosheath ion energies $(\approx 100 \mathrm{eV})$. This suggests significant deceleration of ions upon their entry into the magnetosphere at high latitudes in the mantle region (Newell et al., 1991).

Interball AP measurements confirm that the cusp near 12:00 MLT is also a significant source of outflowing ionospheric ions, as observed in Fig. 8. In the most equatorward part of the cusp, where the strongest fluxes of precipitating electrons are recorded from about 00:18 


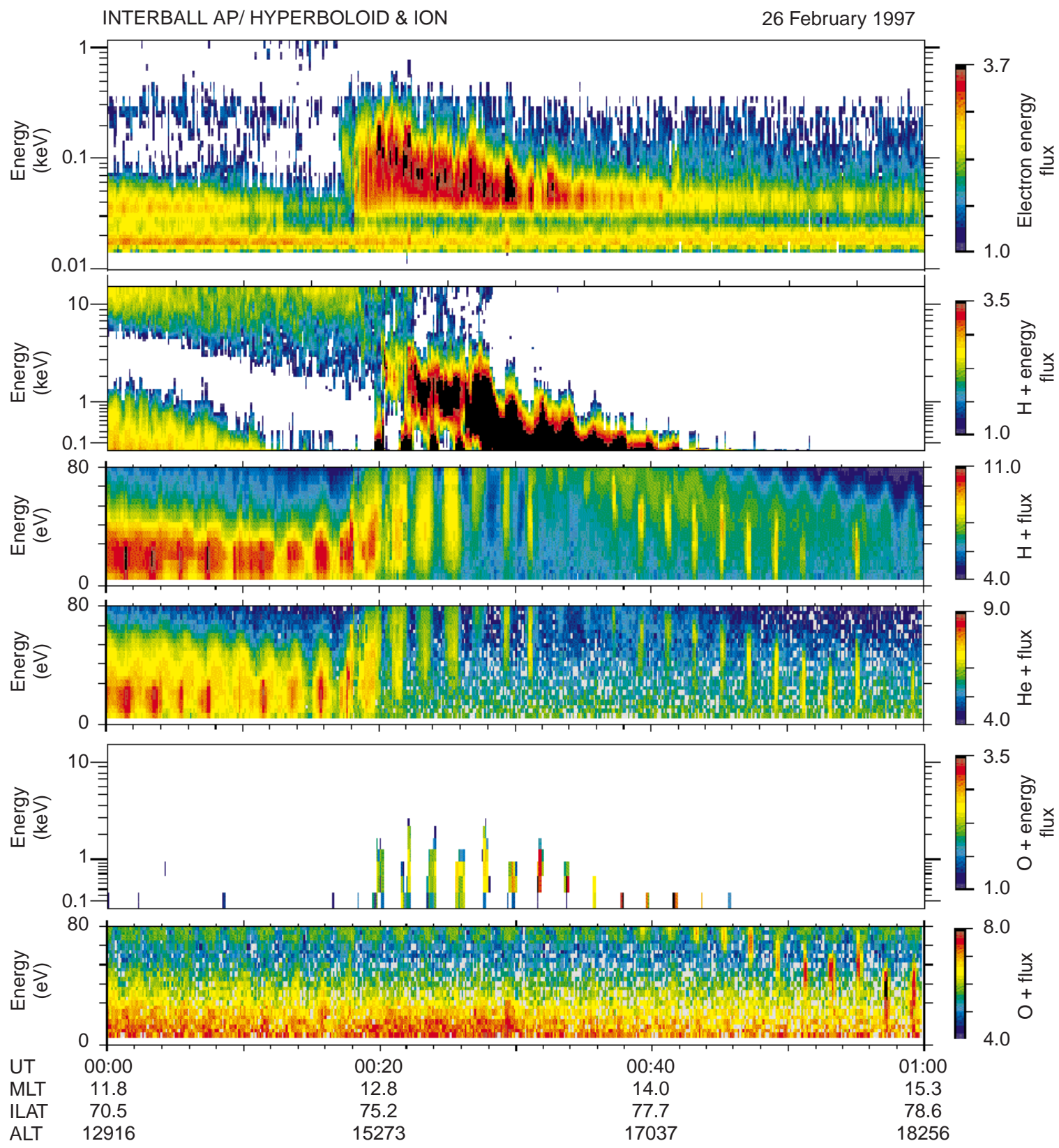

Fig. 8. Energy-time spectrograms of particles during a cusp crossing on 26 February 1997. From top to bottom, $10 \mathrm{eV}-1 \mathrm{keV}$ electrons, $100 \mathrm{eV}-14 \mathrm{keV} \mathrm{H}^{+}$ions, $0-80 \mathrm{eV} \mathrm{H}^{+}$ions, $0-80 \mathrm{eV} \mathrm{He}^{+}$ions, $100 \mathrm{eV}-14 \mathrm{keV} \mathrm{O}^{+}$ions, and $0-80 \mathrm{eV} \mathrm{O}^{+}$ions. High-energy

measurements in $\log$ (counts) are from the Ion experiment. Lowenergy fluxes from Hyperboloid in $\log \left[\left(\mathrm{m}^{2} \mathrm{~s} \mathrm{sr} \mathrm{eV}\right)^{-1}\right]$ are averaged over Hyperboloid half-planar field of view

UT to 00:32 UT Hyperboloid data show upflowing $\mathrm{H}^{+}$ and $\mathrm{He}^{+}$ion distributions identified at each satellite spin. The fluxes are widely spread both in time (i.e., in azimuth angle) and in energy, indicating strong ion heating. $\mathrm{H}^{+}$and $\mathrm{O}^{+}$upflowing fluxes are in fact registered at energies up to a few $\mathrm{keV}$ by the Ion experiment. During this period, strong fluxes are measured at pitch angles down to $90^{\circ}$ (pitch-angle data not shown), suggesting that ions are accelerated perpendicular to the magnetic field over a wide range of altitudes below the satellite. Parallel electrostatic potential drops might also contribute to the acceleration process, but are not required (Crew et al., 1990; Lundin and Eliasson,
1991). Indeed, perpendicular ion acceleration has been observed by the Sounding of the Cleft Ion Fountain Energization Region (SCIFER) rocket experiment down to very low altitudes in the cusp region (Kintner et al., 1996).

Another region can be identified in Fig. 8 from about 00:34 UT. The $\mathrm{H}^{+}, \mathrm{He}^{+}$, and at later times $\mathrm{O}^{+}$ distributions observed by Hyperboloid become narrower, indicating smaller temperatures. All three species exhibit a gradual decrease in energy while the satellite moves at higher altitude toward the polar cap. The data are consistent with the mass spectrometer effect associated with the "cleft ion fountain" (Lockwood et al., 


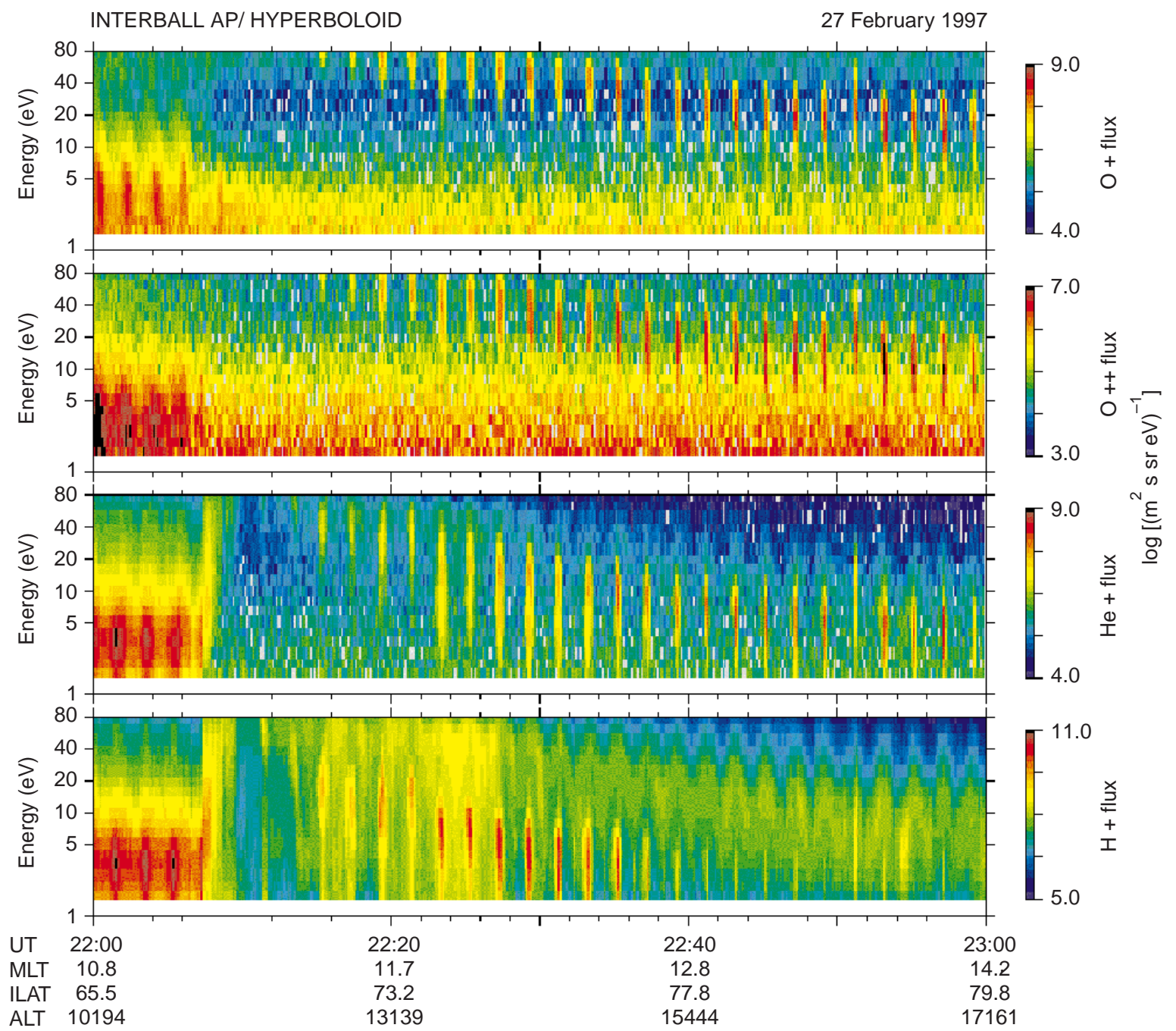

Fig. 9. Energy-time spectrograms for low-energy ions measured by Hyperboloid, during a cusp/cleft crossing on 27 February 1997 . From top to bottom, $\mathrm{O}^{+}, \mathrm{O}^{++}, \mathrm{He}^{+}$, and $\mathrm{H}^{+}$fluxes averaged over Hyperboloid half-planar field of view

INTERBALL AP / HYPERBOLOID
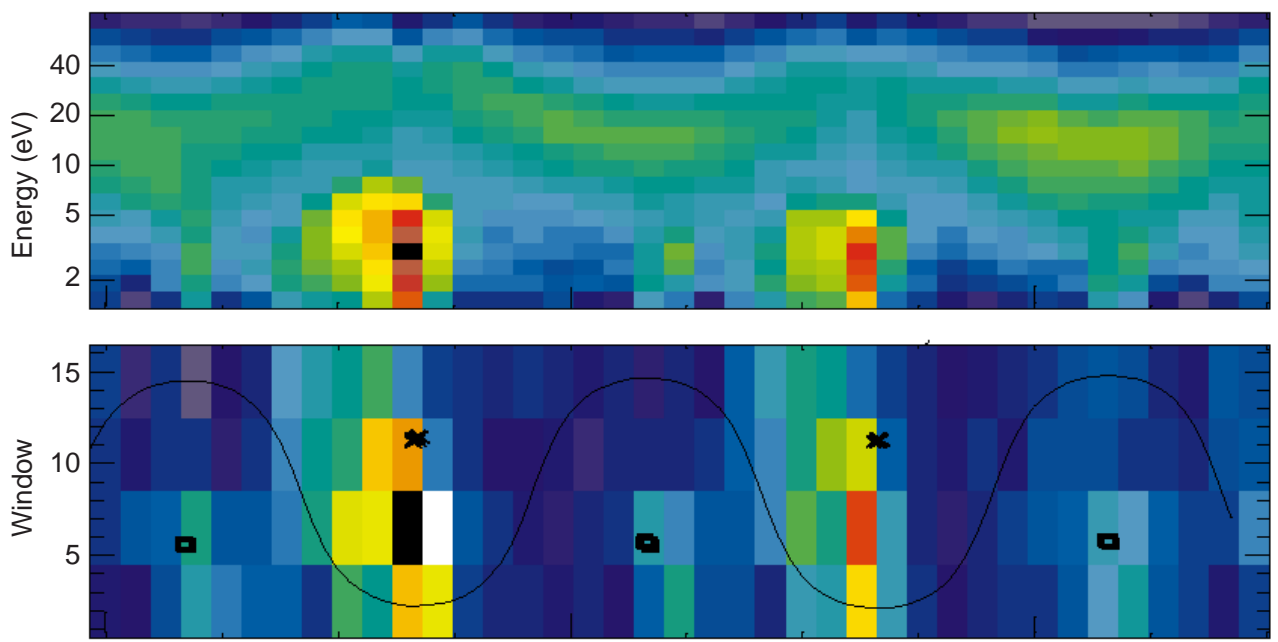

UT $22: 34$

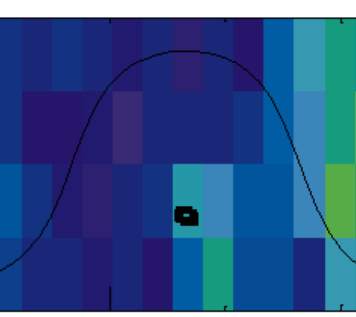

$22: 36$
27 February 1997

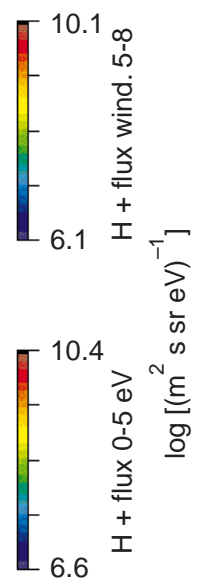

22:38

Fig. 10. Energy-time spectrogram for windows 5 to 8 of the A16 analyzer (top panel) and window-time spectrogram for energies below 5 eV (bottom panel) obtained for $\mathrm{H}^{+}$ions on 27 February 1997 between 22:34 and 22:38:30 UT 
1985). Depending on their velocity, ions are swept by the antisunward convection up to different invariant latitudes during their upward motion to the satellite. Therefore, both energy and mass dispersion occurs for these ions, as evidenced on Fig. 8. The heavier $\mathrm{O}^{+}$ions are convected over large distance away from the source region, which suggests that a great part of the $\mathrm{O}^{+}$ions observed in the polar cap originates in the polar cleft.

Figure 9 shows another example, from 27 February 1997. On that day, the low-latitude edge of the cusp was located as far south as $69^{\circ}$ invariant latitude (IMF conditions were $B_{y}=-5 \mathrm{nT}, B_{z}=-13 \mathrm{nT}$ ). Interball AP enters the cusp at 22:08 UT. Again, this boundary appears as a region of strong ion acceleration. Between 22:07 UT and 22:09 UT, fluxes at energies above 12-eV peak at pitch angles near $90^{\circ}$ (pitch-angle data not shown), indicating a near local perpendicular acceleration. Besides the magnetosheath $\mathrm{H}^{+}$ion injection, the main feature is again the occurrence of strong outflow for all ion species. Moment calculations provide similar upward velocities for all four ion species, e.g., around $22: 31 \mathrm{UT}, v_{/ /} \approx-20 \mathrm{~km} \mathrm{~s}^{-1}$, for an horizontal velocity $\approx 8 \mathrm{~km} \mathrm{~s}^{-1}$ mainly due to convection. The data thus provide a quantitative confirmation of the cleft ion fountain mechanism.

Another interesting and surprising phenomenon is the presence, in the interval 22:20-22:50 UT, of a very narrow and low energy $(<5 \mathrm{eV}) \mathrm{H}^{+}$population detected between the upflowing ion events. This signature is more clearly seen in Fig. 10, which represents a zoom for the interval 22:34-22:38:30 UT. Note the enhanced fluxes occurring in the second window group between 2 and $5 \mathrm{eV}$ at times 22:34:20, 22:36:30, and 22:38:25 UT. These fluxes were indeed detected nearly along (downward) the local magnetic field direction (bottom panel). These ions seem clearly separated from the V-shaped injected ions at higher energies (top panel), and are therefore probably not magnetosheath ions. If they are of ionospheric origin, they have energies too high to be gravitationally bounded. Their downward motion might be the trace of small amplitude, downward pointing DC electric fields above the satellite altitude,

\section{Conclusion}

Since its first switching-on and commissioning phase at the end of September 1996, the Hyperboloid experiment has been working nominally. In more than one year of operation, full ion distribution functions in the $0-80-\mathrm{eV}$ range, and with mass separation, have been obtained at altitudes between $\approx 10000$ and $20000 \mathrm{~km}$ in the outer plasmasphere, auroral zone, and polar-cap regions, with full MLT coverage. More recently, the experiment has been operated during the short $(<14 \mathrm{~min})$ perigee passes below the radiation belts, between $\approx 700$ and $2000 \mathrm{~km}$ altitudes, again at auroral and polar latitudes. A great variety of phenomena has been observed. Hyperboloid detailed distribution function measurements offer new perspectives for understanding process- es, such as polar wind or the cleft ion fountain, which were already identified during previous mission (DE, Akebono, Viking). New observations, e.g., $\mathrm{H}^{+}$and $\mathrm{O}^{+}$ conics at energies $\approx \mathrm{a}$ few tens of eVs observed simultaneously at altitudes $\approx 3$ Earth's radii, or thermal ion signatures of the polar edge of the discrete auroral oval, deserve further experimental investigation and are expected to give rise to new theoretical developments.

Data acquired in the following lifespan of the experiment will improve the statistical basis of future studies. Whenever possible, use will be made of magnetic conjugations with other satellites and groundbased radars. Finally, particular attention will be paid to modeling the interaction of the satellite and of the experiment with their plasma environment, in order to improve the physical significance of the data.

Acknowledgements. The Interball Project was accomplished in the frame of contract N025-7535/94 with the Russian Space Agency (RKA). The work of Y.G., D.C., T.M., and L.Z. was supported by grants from Russian Foundation for Basic Research (RFBR) N 94-02-04299 and 97-02-16333, and by grant INTAS N 94-1695. We thank Russian Space Agency, Lavochkin Space Association and Babakin Space Center for managing the different steps of the project with success despite very adverse conditions. The experiment was financially supported by CNES under the auspices of grants covering the period 1985 to 1998 . We are indebted to J.-A. Sauvaud and S. Perraut for providing data from the Ion and IESP experiments. Thanks are also due D. Delcourt for fruitful discussions.

Topical Editor K.-H. Glassmeier thanks K. Torkar and R. Lundin for their help in evaluating this paper.

\section{References}

Abe, T., B. A. Whalen, A. W. Yau, R. E. Horita, S. Watanabe, and E. Sagawa, EXOS D (Akebono) suprathermal mass spectrometer observations of the polar wind, J. Geophys. Res., 98, 11191, 1993.

André, M., P. Norqvist, A. Vaivads, L. Eliasson, O. Norberg, A. I. Eriksson, and B. Holback, Transverse ion energization and wave emissions observed by the Freja satellite, Geophys. Res. Lett., 21, 1915, 1994.

Banks, P. M., and T. E. Holzer, High-latitude plasma transport: the polar wind, J. Geophys. Res., 74, 6317, 1969.

Berthelier, J.-J., J. Covinhes, M. Godefroy, G. Gogly, C. Guérin, D. Roux, P. Thévenet, and V. A. Gladyshev, The thermal ion mass spectrometer on board Aureol-3: the DYCTION experiment, Ann. Geophys., 38, 591, 1982.

Burch, J. L., P. H. Reiff, R. A. Heelis, J. D. Winningham, W. B. Hanson, C. Gurgiolo, J. D. Menietti, R. A. Hoffman, and J. N. Barfield, Plasma injection and transport in the mid-altitude polar cusp, Geophys. Res. Lett., 9, 921, 1982.

Calvert, W., Auroral plasma cavity, Geophys. Res. Lett., 8, 720, 1981.

Cladis, J.B., Parallel acceleration and transport of ions from polar ionosphere to plasma sheet, Geophys. Res. Lett., 13, 893, 1986.

Crew, G. B., T. Chang, J. M. Retterer, W. K. Peterson, D. A. Gurnett, and R. L. Huff, Ion cyclotron resonance heated conics: theory and observations, J. Geophys. Res., 95, 3959, 1990.

Feldstein, Y. I, and Y. I. Galperin, The auroral luminosity structure in the high-latitude upper atmosphere: its dynamics and relationship to the large-scale structure of the Earth's magnetosphere, Rev. Geophys. Space Phys., 23, 217, 1985.

Galperin, Y. I., and Y. I. Feldstein, Auroral luminosity and its relationship to magnetospheric plasma domains, in Auroral 
physics, Eds. C.-I. Meng, M. J. Rycroft, and L. A. Frank, Cambridge UP, Cambridge, p. 207, 1991.

Galperin, Y. I., and Y. I. Feldstein, Mapping of the precipitation regions to the plasma sheet, J. Geomagn. Geoelectr., 48, 857, 1996.

Ganguli, S. B., The polar wind, Rev. Geophys., 34, 311, 1996.

Giles, B. L., C. R. Chappell, T. E. Moore, R. H. Comfort, and J. H. Waite, Jr., Statistical survey of pitch angle distributions in core $(0-50 \mathrm{eV})$ ions from Dynamics Explorer 1: outflow in the auroral zone, polar cap, and cusp, J. Geophys. Res., 99, 17483, 1994.

Green, J. L., and J. H. Waite, Jr., On the origin of polar ion streams, Geophys, Res. Lett., 12, 149, 1985.

Gurgiolo, C., and J. L. Burch, DE-1 observations of the polar windA heated and an unheated component, Geophys. Res. Lett., 9, $945,1982$.

Gustafsson, G., M. André, L. Matson, and H. Koskinen, On waves below the local proton gyrofrequency in auroral acceleration regions, J. Geophys. Res., 95, 5889, 1990

Heikkila, W. J., and J. D. Winningham, Penetration of magnetosheath plasma to low altitudes through the dayside magnetospheric cusps, J. Geophys. Res., 76, 883, 1971.

Ho, C. W., J. L. Horwitz, and G. R. Wilson, Dynamics of the $\mathrm{H}^{+}$ and $\mathrm{O}^{+}$polar wind in the transition region as influenced by ionospheric convection and electron heating, J. Geophys. Res., 102, 395, 1997.

Hoffman, J. H., Studies of the composition of the ionosphere with a magnetic deflection mass spectrometer, Int. J. Mass Spectrom. Ion Phys., 4, 315, 1970.

Hoffman, J. H., W. H. Dodson, C. R. Lippincott, and H. D. Hammack, Initial ion composition from the ISIS 2 satellite, $J$. Geophys. Res., 79, 4246, 1974.

Horwitz, J. L., and M. Lockwood, The cleft ion fountain: a twodimensional kinetic model, J. Geophys. Res., 90, 9749, 1985.

Horwitz, J. L., S. Menteer, J. Turnely, J. L. Burch, J. D. Winningham, C. R. Chappell, J. D. Craven, L. A. Frank, and D. W. Slater, Plasma boundaries in the inner magnetosphere, $J$. Geophys. Res., 91, 8861, 1986.

Kintner, P. M., J. Bonnell, R. Arnoldy, K. Lynch, C. Pollock, T. Moore, J. Holtet, C. Deehr, H. Stenbaek-Nielsen, R. Smith, J. Olson, and J. Moen, The SCIFER experiment, Geophys. Res. Lett., 23, 1865, 1996.

Klumpar, D. M., W. K. Peterson, and E. G. Shelley, Direct evidence for two-stage (bimodal) acceleration of ionospheric ions, $J$. Geophys. Res., 89, 10779, 1984.

Kremser, G., and R. Lundin, Average spatial distributions of energetic particles in the mid altitude cusp/cleft region observed by Viking, J. Geophys. Res., 95, 5753, 1990.

Le Quéau, D., A. Roux, J.-L. Rauch, F. Lefeuvre, and J.-M. Bosqued, Heating of protons by resonant absorption in a multicomponent plasma, 2. Theoretical model, J. Geophys. Res., 98, 13363, 1993.

Lockwood, M., M. O. Chandler, J. L. Horwitz, J. H. Waite, Jr., T. E. Moore, and C. R. Chappell, The cleft ion fountain, $J$. Geophys. Res. 90, 9736, 1985.

Louarn, P., J. E. Wahlund, T. Chust, H. de Feraudy, A. Roux, B. Holback, P. O. Dovner, A. I. Eriksson, and G. Holmgren, Observations of kinetic Alfvèn waves by the Freja spacecraft, Geophys. Res. Lett., 21, 1847, 1994.

Lundin, R., and L. Eliasson, Auroral energization processes, Ann. Geophysicae, 9, 202, 1991.

Lundin, R., G. Gustafsson, A. I. Eriksson, and G. Marklund, On the importance of high-altitude low-frequency electric fluctuations for the escape of ionospheric ions, J. Geophys. Res., 95, 5905, 1990

Menietti, J. D., and J. L. Burch, Spatial extent of the plasma injection region in the cusp-magnetosheath interface, J. Geophys. Res., 93, 105, 1988.

Moore, T. E., C. R. Chappell, M. O. Chandler, S. A. Fields, C. J. Pollock, D. L. Reasoner, D. T. Young, J. L. Burch, N. Eaker, J. H. Waite, Jr., D. J. McComas, J. E. Nordholdt, M. F. Thomsen,
J.-J. Berthelier, and R. Robson, The thermal ion dynamics experiment and plasma source instrument, Space Sci. Rev., 71, 409, 1995.

Mozer, F. S., On the lowest altitude S3-3 observations of electrostatic shocks and parallel electric fields, Geophys. Res. Lett., 7, 1097, 1980.

Nagai, T., J. H. Waite, Jr., J. L. Green, C. R. Chappell, R. C. Olsen, and R. H. Comfort, First measurements of supersonic polar wind in the polar magnetosphere, Geophys. Res. Lett., 11, 669, 1984.

Newell, P. T., and C. I. Meng, The cusp and the cleft/boundary layer: low-altitude identification and statistical local time variation, J. Geophys. Res., 93, 14549, 1988.

Newell, P. T., W. J. Burke, C.-I. Meng, E. R. Sanchez, and M. E. Greenspan, Identification and observations of the plasma mantle at low altitude, J. Geophys. Res., 96, 35, 1991.

Newell, P. T., Y. I. Feldstein, Y. I. Galperin, and C.-I. Meng, The morphology of nightside precipitation, J. Geophys. Res., 101, 10737, 1996.

Perraut, S., ULF wave measurements on board the Interball Auroral probe, Ann. Geophysicae, this issue, 1998.

Pollock, C. J., M. O. Chandler, T. E. Moore, J. H. Waite, Jr., C. R. Chappell, and D. A. Gurnett, A survey of upwelling ion event characteristics, J. Geophys. Res., 95, 18969, 1990.

Rauch, J.-L., F. Lefeuvre, D. Le Quéau, A. Roux, J.-M. Bosqued, and J.-J. Berthelier, Heating of protons by resonant absorption in a multicomponent plasma, 2. Experimental evidence, $J$. Geophys. Res., 98, 13347, 1993.

Reiff, P. H., T. W. Hill, and J. L. Burch, Solar wind plasma injection at the dayside magnetospheric cusp, J. Geophys. Res., 82, 479, 1977.

Retterer, J. M., T. Chang, and J. R. Jasperse, Transversely accelerated ions in the topside ionosphere, J. Geophys. Res., 99, 13189, 1994

Sauvaud, J.-A., The Ion experiment on board the Interball Auroral satellite: initial results on velocity dispersed structures in the cleft and inside the auroral oval, Ann. Geophysicae, this issue, 1998.

Schunk, R. W., and J. J. Sojka, A three-dimensional timedependent model of the polar wind, J. Geophys. Res., 94, 8973, 1989.

Shelly, E. G., R. G. Johnson, and R. D. Sharp, Satellite observations of energetic heavy ions during a geomagnetic storm, J. Geophys. Res., 77, 6104, 1972.

Shelly, E. G., A. G. Ghielmetti, H. Balsiger, R. K. Black, J. A. Bowles, R. P. Bowman, O. Bratschi, J. L. Burch, C. W. Carlson, A. J. Coker, J. F. Drake, J. Fischer, J. Geiss, A. Johnstone, D. L. Kloza, O. W. Lennartsson, A. L. Magoncelli, G. Paschmann, W. K. Peterson, H. Rosenbauer, T. C. Sanders, M. Steinacher, D. M. Walton, B. A. Whalen, and D. T. Young, The toroidal imaging mass-angle spectrograph (TIMAS) for the polar mission, Space Sci. Rev., 71, 497, 1995.

St-Maurice, J.-P., and R. W. Schunk, Ion velocity distribution in the high-latitude ionosphere, Rev. Geophys., 17, 99, 1979.

Taylor, H. A., Jr, and W. J. Walsh, The light ion trough, the main trough, and the plasmapause, J. Geophys. Res., 77, 6716, 1972.

Temerin, M., and R. L. Lysak, Electromagnetic ion cyclotron mode (ELF) waves generated by auroral electron precipitation, $J$. Geophys. Res., 89, 2849, 1984.

Torkar, K., An experiment to study and control the Langmuir sheath around Interball-2, Ann. Geophys., this issue, 1998.

Ungstrup, E., D. M. Klumpar, and W. J. Heikkila, Heating of ions to suprathermal energies in the topside ionosphere by electrostatic ion cyclotron waves, J. Geophys. Res., 84, 4289, 1979.

Watanabe, S., B. A. Whalen, and A. W. Yau, Thermal ion observations of depletion and refilling in the plasmaspheric trough, J. Geophys. Res., 97, 1081, 1992.

Zelenyi, L. M., R. A. Kovrazhkin, and J.-M. Bosqued, Velocitydispersed ion beams in the nightside auroral zone: AUREOL-3 observations, J. Geophys. Res., 95, 12119, 1990. 